\title{
Driving Simulator
}

\author{
Ambar Yadav ${ }^{1}$, Arti Singh ${ }^{2}$ \\ 1,2(Wireless Communication Networks, Gautam Buddha University, Greater Noida-India)
}

\begin{abstract}
Simulators which are developed by suppliers individually for specific application purpose are known as Driving Simulator. Most of the conventional driving simulators provide some flexibility for constructing new test scenarios, but do not allow users to change system arrangement. Driving simulator structure are rather complex but it provide user friendly environment. This paper describes the concept and main idea of about driving simulator. Some common DS like CAR SIM and Truck SIM and their Mathematical models. What about Virtual Environment in DSand finally the advantages and disadvantages of DS.
\end{abstract}

Keywords: CAR SIM, TRUCKSIM, DS, Advance driving assistance system

\section{Introduction}

Simulate is the word that have same meaning as fabricate, feign, pretend, copy, mimic, or imitate. The word "simulation" can be defined as a "technique of substituting a real environment to fake one, so that it is possible to work under laboratory conditions of control. Within experimentally controlled environment, performance measures can be defined, collected, and repeatly tested which is in a cost-effective manner (Olsen 1996).

A virtual driving simulator is a device that allows user to feel a life-like experience of driving an actual vehicle within virtual reality. It is effectively used for studying the interaction of a driver and vehicle and for developing new vehicle systems, human factor study, and vehicle safety research by enabling the reproduction of the actual driving environments in a safe and tightly controlled environment (Kang et al. 2004). Mostly vehicle simulators consist of physical mockups as examples steering wheel, gearshift and pedals. These are essential in trying to simulate real conditions, then it's become as a drawback for system becomes more expensive, more huge (non mobile), and then limited to reflect changes on the vehicle type, dimensions, or interior design (Kallmann et al. 2003).

The virtual driving simulator environment consists of static universe, dynamic objects and interior of driver's vehicle (Kang et al. 2004). The static universe can be building, trees, road and others. The dynamics objects can include any moving objects in virtual scene like cars, people, and crowd. With more complex virtual scene will contain many thousands of polygons which need more graphic processing power and more computation cost to render the scene. Even on latest graphic hardware processing, the increase in complexity in virtual environment will increase computational power. The complex scene is needed to manage so it can be efficiently render in real time and avoid memory leak. Implement of $3 \mathrm{~d}$ scene graphic management techniques help to reduce computational burden in complex driving simulator environment. Figure 1 shows an example of a driving simulator and complex scene in driving simulator virtual environment [1]

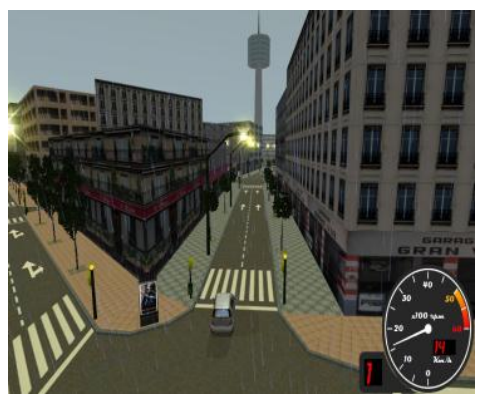

Fig. 1The complex virtual environment of driving simulator

Most simulators act in isolation from the real world, so when we see one that breaks out of its virtual shell, we're more than a little intrigued. Saginomiya has designed a driving simulator that's directly linked to an actual car's components sitting on a six-axis motion system. The whole affair works as a form of virtuous circle for testing, where the real parts feed on the simulator and vice versa: since the simulator is based on the internal model of the car, it can translate road results directly to the suspension and steering of the physical components, which promptly loop around and dictate force feedback in the simulator through actuators in the steering wheel. Apart from creating what amounts to the real real driving simulator, Saginomiya's invention is a huge boon to 
automakers, which can test how key components work without having to build the whole vehicle first. Sadly, the simulator likely won't reach full-on Avatar levels and steer a car on a real road anytime soon, but it's engaging enough that we can see more than a few test drivers putting in overtime just for kicks.

Simulators similar to the one recently unveiled to the world by Toyota aren't all that uncommon, but this 4.5meter high pod is "the first to move laterally and has the longest range of 35-meters front to back and 20-meters from right to left." Reportedly, the automaker plans to use its newest toy to "analyze driving characteristics under various conditions such as drunkenness and drowsiness" and subsequently decide what new safety features should be added to its fleet of motorcars. More specifically, the device enables researchers to "conduct driving tests that would be too dangerous to perform in the real world or that require specific driving conditions," and we're pretty sure the 360-degree concave video screen is quite the draw, too. Unfortunately, we have little faith that you'll ever see a tamed version of this here simulator at your local arcade, but one can hope, we suppose.

\section{TYPES OF SIMULATOR}

Simulators can be divided as interactive and non-interactive. Interactive driving simulator mean that the simulator responds to the driver just as the driver responds to the simulator or called as a closed-loop driving simulator. Apart in a non-interactive system, the driver responds to the simulator, but the simulator does only what it was programmed to do, regardless of the driver's actions, often referred to as an open-looped system. Interactive driving simulation represents real world such as actual roadways through computer generated imagery (CGI), auditory feedback, and realistic vehicle instruments and controls such as brake pedal, steering wheel, turn signal indicator, speedometer, and mirrors.

CarSim and TruckSim are being used in a wide variety of driving simulators. These range in size and price, from $\$ 20,000$ Desktop systems to $\$ 100,000,000$ full-vehicle simulators. If you want a low-cost turnkey driving simulator from Mechanical Simulation, you can get CarSim DS or TruckSim DS.

CarSim DS-CarSim DS is a software for simulating and analyzing the behavior of four-wheeled vehicles in response to steering, braking, and acceleration inputs. CarSim runs faster than real-time using ordinary PC's. The basic CarSim packages require no other software.It is fully compatible with other simulation environments like MATLAB/Simulink, LabView and programming languages such as Visual Basic and C.

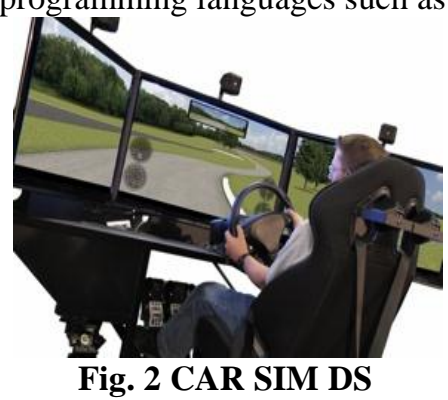

\section{CarSim Math Models}

- Control Inputs

- Environmental Inputs

- 3D Vehicle Dynamics

- Tires

The three main control inputs are steering, braking, and speed.

It has options for open-loop and closed-loop inputs for steering, braking, throttle, and gear shifting. It has also options for a new control which can be triggered by any output variable reaching a specified value.

\section{Environmental Inputs Model}

The main environmental input is the 3D road surface. In this model roads can be build with arbitrary horizontal, vertical, and cross-elevation geometry. Friction between the tire and the ground is specified as a function of distance along the road and lateral position relative to the centerline. The CarSim graphical user interface (GUI) also provides a road building tool for rapidly assembling a 3D road surface from sections. Besides providing the $3 \mathrm{D}$ road information to the math models, the GUI also generates shapes for the CarSim animator automatically. In this regard, the 3D surface can always be visualized.

CarSim also includes aerodynamic effects and wind inputs.

\section{D Vehicle Dynamics Model}

The equations of motion used are for full nonlinear 3D motions of rigid bodies. The major kinematics and compliance effects of the suspensions and steering systems are specified with properties which can be 
measured directly. Details of the linkages and gears in the suspensions and steering systems are not needed, reducing the amount of information needed to obtain accurate predictions. Many of the components that have the greatest effect on handling, braking, and acceleration are represented with nonlinear tables of measurable data. For example, CarSim uses detailed nonlinear kinematical relationships, nonlinear spring models, and other nonlinear component models.

\section{Tires Model}

The CarSim tire models reproduce the shear forces and moments applied to the tires from the 3D road surface. They provide the full nonlinear behavior of rolling tires subject to combined braking/acceleration and cornering on roads with variable friction. The models can handle the transmission to zero speed to properly simulate parking conditions. CarSim includes several tire models with alternate ways to provide the entire tire data.

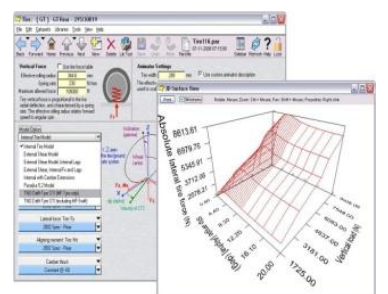

\section{TRUCK SIM}

Fig. 3 TIRE MODEL

TruckSim is a software package for simulating and analyzing the behavior of heavy trucks and combination vehicles in response to steering, braking, and acceleration inputs. The truck models and some of the smaller combination vehicle models run faster than real-time using ordinary PC's. The basic TruckSim packages require no other software, although full compatibility with other simulation environments such as MATLAB/Simulink, LabView, and ETAS ASCET is included for users who want to extend the models. Additional packages offer extra capabilities.

TruckSim includes the VehicleSim (VS) browser and database, engineering plotter, animator, and vehicle models. The vehicle models are provided as dynamically linked libraries (DLL's). The same DLL is used for stand-alone runs (the core vehicle model running with no external software), or with Simulink, or LabView, or ASCET, or custom programs written in C. The DLL always runs at high speed, giving results much faster than real-time on typical PCs. Of course, identical behavior is obtained regardless of the simulation environment, because there is just one DLL used in different contexts.

Extended TruckSim models are available with mounted cabs and frame torsional compliance. The cab mounts are important when evaluating ride quality in the cab. The frame torsional compliance is an important factor in determining the load transfer to the tires and therefore it affects handling and other behaviors.

The extra complexity slows the simulation time by about $20 \%$ and requires data that might be hard to obtain for many users; this is why the basic models are based on the more simple assumption of torsionally rigid frames. The sensor option provides up to 20 range and detection sensors. The sensors provide 11 variables for each detected object (range, range rate of change, bearing angles, elevation, magnitude, etc.). The detection variables are typically sent to Simulink for use in user-defined advanced driving assistance systems (ADAS) or other advanced controllers. The sensors have sensitivity to range, bearing, elevation, and reflectiveness. Targets can be rectangular orcylindrical. Occlusion (blocking of view) is supported, to simulated conditions where some objects block others, or when part of a large object (building, parked car, etc.) is partly outside the range of the sensor field. TruckSim has an additional option for low-level integration with the commercial power train simulation software Cruise from AVL. With this option, the internal power train model in TruckSim is replaced with a model from AVL Cruise.

\section{ENVIRONMENT IN DRIVING SIMULATOR}

The virtual environment (VE) will includes full texturing, shading, fog, and lighting effects with an aim to stimulate a real driving environment.[10] The development of the $3 \mathrm{D}$ model is the most difficult and interesting component of the simulator. The visual database of the simulator can be divided into three types of Three dimensional models are (Liao D. 2006).

[1]. 3-D Street Models- Includes terrain, tree, buildings and traffic devices models.

[2]. 3-D roadway Models- Modelled separately to allowed collusion detection between road surface and vehicle.

[3]. 3-D mobile Models- The vehicles modelled so can be able to create the feel inside real driving simulator. 


\section{DELAY IN GRAPHICS RESULTS"SIMULATOR SICKNESS"}

Simulator sickness was firstly documented by Havron and Butler in 1957 in a helicopter trainer. It almost similar to motion sickness, but can occur without actual motion of the subject. Simulator sickness is potential treat that will cause severe discomfort some user during or after using driving simulator (Kolasinski 1995). It is one of the biggest problems that faced many driving simulators. Two factors can probably causes simulator sickness are transport delay (time lag) and update rate (frame rate). Transport delay is a problem whereby the response of a dynamic element slower in time relative to its input (Lee et al. 1998).

Three major sources of transport delay in the driving simulator: the vehicle simulation, visual, and motion systems. Real time vehicle simulation is related with vehicle dynamics computation remains lower than the perception threshold of the driver. Next, in the visual system includes data acquisition, image processing and display time (is in the range of 25 and $50 \mathrm{msec}$ depending on the quality of images). Update rate also known as frame rate is can be defined as speed of simulation. This frame rate is different the refresh rate where it depend on the scene complexity and graphic processing power of hardware used by the driving simulators. The effects of this problem will cause visual lag. Frame rate can be referred as benchmark. When render the scene with high complexity, the frame rate will drop.

With these two factors the solution needed to minimize the problems which may be not lead directly to simulator sickness problem. The important is to ensuring the high fidelity in complex driving simulator virtual environment.

\section{Advantages of driving simulators}

Driving simulators offer various advantages, including:

1. Controllability, reproducibility, and standardization. [13] Behavior of virtual traffic, weather conditions, and the road layout can be manipulated (offline or in real-time) as a function of the training needs or research aims. Purpose-developed scenarios enable trainees to practice a large number of dedicated maneuvers per time unit, describe software architecture for generating dynamic scenarios in a driving simulator. With the aim of maximizing the effectiveness of the training, the authors apply a metaphor from the 1998 movie The Truman Show: everything surrounding the learner driver responds to the driver's behavior. Using simulators, participants in different physical locations can drive under the exact same conditions. This is beneficial for creating standardized driving tests and reproducible research results. In contrast, the real traffic environment is largely random.

2. Ease of data collection. A driving simulator can measure performance accurately and efficiently. With a real vehicle, it is far more cumbersome to obtain complete, synchronized, and accurate measurement data. It is a fundamental challenge to get an accurate recording of where a real vehicle actually is in the world. For example, in one study using an instrumented vehicle and a driving simulator, it was impossible to determine the distance between the vehicle and a stop line on the road, while in the simulator this information was readily available [5]. Measurement of lateral position is challenging as well, as this requires visible lane markers while weather conditions, reflection, and shades may affect the quality of the measurement [6]. Santos et al. [7] found that lateral position measurements of the instrumented vehicle were of marginal quality while this information was accurate in the simulator, leading the authors to conclude that "problems with field studies in an instrumented vehicle have been confirmed". Because of the measurement capabilities of simulators, new types of behavior analyses come within reach, such as trigonometric analysis of time-to-line crossing [8] or object detection and hazard perception research using eye-tracking [9].

3. Possibility of encountering dangerous driving conditions without being physically at risk. Simulators can be used to prepare trainees to handle unpredictable or safety-critical tasks that may be inappropriate to practice on the road, such as collision avoidance or risky driving [10]. In addition, simulators make it possible to study hazard anticipation and perception by exposing drivers to dangerous driving tasks, which is an ethically challenging endeavor in real vehicles [9]. [11] Stated that simulators "offer an opportunity to learn from mistakes in a forgiving environment" (p. 134). Allen et al. [12] made a similar case: "Motor vehicle crashes are significantly higher among young drivers during the first year of licensure, and crash risks decline with increased experience. This produces an interesting dilemma about how to provide young drivers with driving experience without significantly increasing their crash risk. Driving simulation may be the solution to this dilemma."

4. Novel opportunity for feedback and instruction. Simulators offer the opportunity for feedback and instruction that is not easily achieved in real vehicles. For example, it is possible to freeze, reset, or replay a scenario [13]. Feedback and instructions can also be delivered in other modalities besides speech, such as visual overlays to highlight critical features in the environment. 


\section{Disadvantages of driving simulators}

However, simulators have several known disadvantages and challenges, including:

1. Limited physical, perceptual, and behavioral fidelity. Low-fidelity simulators may evoke unrealistic driving behavior and therefore produce invalid research outcomes. Simulator fidelity is known to affect user opinion. Participants may become demotivated by a limited-fidelity simulator and prefer a real vehicle instead (or a more costly high-fidelity simulator for that matter). Interestingly, while safety is often cited as an advantage of driving simulation (see above), sometimes this same feature is interpreted as a disadvantage. For example, Käppler [13] pointed out that real danger and the real consequences of actions do not occur in a driving simulator, giving rise to a false sense of safety, responsibility, or competence. Simply investing resources to increase fidelity is not necessarily a desirable solution, as it adds to the complexity of the device and might hamper experimental control. In some cases, deliberate deviations from reality yield valid results.

2. Shortage of research demonstrating validity of simulation. A growing body of evidence indicates that drivingsimulator measures are predictive for on-the-road driving performance. However, only a few studies have investigated whether skills learned in a driving simulator transfer to the road. Note that in the field of aviation, studies on the transfer of training are far more common, but even in aviation critical questions remain unanswered, for example whether a motion base provides added value for the effectiveness of flight training .

3. Simulator discomfort, especially in older people or under demanding driving conditions. Simulator sickness symptoms may undermine training effectiveness and negatively affect the usability of simulators. This is a serious concern, but fortunately, useful technological and procedural guidelines are available to alleviate it. Research shows that simulator sickness is less of a problem for young drivers. Experience shows that limiting the horizontal field of view, avoiding sharp curves or stops during driving, and using short sessions (10 min) with sufficient rest breaks improves or even eliminates simulator sickness.

\section{Conclusion}

Driving simulator is content a complex virtual environment that needed to manage to make sure efficiently rendered by graphic processing. The first issues discussed are all related to driving simulator: history, type of simulator, architecture of the driving simulator and system design. Part that needed to be highlighted here existence of scene management in driving simulator system design. The next issues discussed are the virtual environment in driving simulator and graphic delay cause simulator sickness. The issue mark here is the increase of complexity will lead to long time lag and decrease frame rate. Last issue discuss is about what is 3D scene management and type of techniques can be used in driving simulator VE. The potentials of scene management in complex scene for driving simulator will lead used to others real time graphic system so complexity of the 3D scene able to manage to achieve fidelity and realism.

\section{References}

[1] Mohd Khalid Mokhtar and MohdShahrizalSunar “3D GRAPHIC SCENE MANAGEMENT IN DRIVING SIMULATOR

[2] Moore, G. E. (1965).Cramming more components onto integrated circuits. Electron 38, 114-117.

[3] Generating dynamic scenarios in a driving simulator. IEEE Intelligent Systems 21, 28-32.

[4] Godley, S. T., Triggs, T.J., Fildes, B.N. (2002). Driving simulator validation for speed research. Accident Analysis and Prevention 34, 589-600.

[5] Roskam, A.J., Brookhuis, K.A., De Waard, D., Carsten, O.M.J., Read, L., Jamson, S., ... Victor, T. (2002). HASTE Deliverable 1: Development of $\quad$ experimental protocol. from 〈http://ec.europa.eu/transport/roadsafety_library/publications/haste_deliverable_1_v1_1.pdf>

[6] Santos, J., Merat, N., Mouta, S., Brookhuis, K., De Waard, D. (2005). The interaction between driving and in-vehicle information systems: Comparison of results from laboratory, simulator and real-world studies. Transportation Research Part F 8, $135-146$.

[7] Van Winsum, W., Brookhuis, K. A., De Waard, D. (2000). A comparison of different ways to approximate time-to-line crossing (TLC) during car driving. Accident Analysis and Prevention 32, 47-56.

[8] Underwood, G., Crundall, D., Chapman, P. (2011). Driving simulator validation with hazard perception. Transportation Research Part F 14, 435-446.

[9] Hoeschen, A., Verwey, W., Bekiaris, E., Knoll, C., Widlroither, H., De Waard, D., et al. (2001).

[10] Flach, J.M., Dekker, S., Stappers, P.J. (2008). Playing twenty questions with nature (the surprise version): Reflections on the dynamics of experience. Theoretical Issues in Ergonomics Science 9, 125-154.

[11] Allen, R.W., Park, G.D., Cook, M.L., Fiorentino, D. (2007). The effect of driving-simulator fidelity on training effectiveness. Proceedings of the Driving Simulation Conference North America, Iowa City, IA.

[12] Vlakveld, W.P. (2005). The use of simulators in basic driver training. HUMANIST Workshop on the application of new technologies to driver training, Brno, Czech Republic. Retrieved from Käppler, W.D. (1993). Views on the role of simulation in driver training. Proceedings of the 12th European Annual Conference on Human Decision Making and Manual Control, Kassel, Germany, 5.12-5.17.

[13] J.C.F. de Winter, P.M. van Leeuwen and R. Happee, "Advantages and Disadvantages of Driving Simulators" 


\section{ABOUT THE AUTHOR}

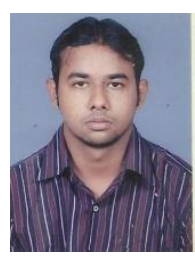
Greater Noida, India. He received his B.Tech. degree in Electronics and Communication (EC) from Integral University, Lucknow, India in 2008. His current research interest includes handoff management protocol for next generation wireless systems, Ubiquitous communication in Next generation wireless systems, adaptive antenna array processing, broad beam forming and their applications.

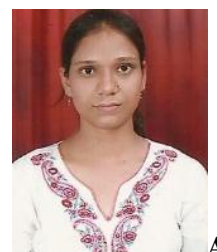
Greater Noida, India. She received her B.Tech Degree in Electronics and Communication (EC) from G.L.A.I.T.M Mathura, India in 2009. She served as a Faculty member in Rameesh Institute of Engineering and Technology(RIET), Greater Noida, during 2011-13.Her current research interests are QoS in Packet Switched Network, Mobile Computing, microstrip antennas, Queue management in VOIP. 